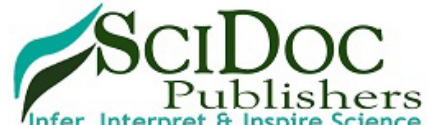

\author{
International Journal of Dentistry and Oral Science (IJDOS) \\ ISSN: 2377-8075
}

\title{
Knowledge, Attitude And Perception On Genomic Markers In Oral Potentially Malignant Disorder Among Oral Medicine And Oral Pathology Specialists
}

Research Article

R. Amritha Sripoo ${ }^{1}$, T. N. Uma Maheswari ${ }^{*}$

${ }^{1}$ Post Graduate Resident, Department of Oral Medicine and Radiology, SIMATS, Chennai, 600007, India.

${ }^{2}$ Professor and Head of the Department of Oral Medicine and Radiology, SIMATS, Chennai, 600007, India.

\section{Abstract}

Background: A genomic biomarker is a measurement of the expression, function and regulation of a gene that consists of one or more deoxyribonucleic acid (DNA) and/or ribonucleic acid(RNA) characteristics. Oral potentially malignant disorders are chronic conditions, which have a higher risk of transformation into oral squamous cell carcinoma.Over years, various specific and non-specific markers have been introduced that could predict the malignant transformation of Oral Potentially Malignant Disorders at early stage. Further,early detection plays a crucial role in prognosis and treatment planning.

Aim: This study aims to evaluate the knowledge, attitude, and perception of genomic markers among oral medicine and oral pathology specialists.

Materials and Method: A 12 multiple choice self constructed questionnaire was prepared by the principal investigator and the guide circulated among peers for validation. An online survey was created using google forms and distributed among Oral Medicine and Oral Pathology Specialists. Data was tabulated in Excel spreadsheets. Imported to IBM SPSS software version 2.0 and statistical analysis were performed. Frequency, percentage and chi square test was performed.

Result: On analysis, it was discovered that Oral medicine and Oral pathology specialists were mostly aware about genomic biomarkers. $42.9 \%$ revealed microRNA 21 as the most sensitive microRNA in OPMD, 49.2\% revealed easy and convenient method for Genomic biomarker research in OPMD as Saliva and 43\% revealed the major difficulty faced during Genomic biomarker research in OPMD is Processing of Samples.Majority of the participants believe that genomic biomarkers can serve as a diagnostic \& prognostic tool. of the participants

Conclusion: Oral medicine and Oral pathology specialists have adequate awareness and knowledge about salivary diagnostic markers, but certain knowledge has to be brushed up among them. Majority of participants showed a positive attitude towards its use as a diagnostic \& prognostic tool. Furthermore, they need to be trained on these grounds to help them treat their patients in the best possible way.

Clinical Significance: There is an impervious need for faster ways to detect different pathologies, seeing that many types of OPMD turning into cancer are discovered only in late stage.miRNAs as a genomic biomarkers can fulfill this, thus being an impressive research field. Further, the identification of biomarkers provides a novel insight into understanding the mechanisms of the tumor formation and progression and promising therapy for different cancers.

Keywords: Knowledge; Attitude; Perception; Biomarkers; Oral Potentially Malignant Disorders; Oral Pathology.

\section{Introduction}

A genomic biomarker is a measurement of the expression, function and regulation of a gene. A genomic biomarker can consist of one or more deoxyribonucleic acid (DNA) and/or ribonucleic acid (RNA) characteristics. Genomic biomarker includes miRNA, RNA, Mt.DNA, DNA[1, 2]. In recent years miRNAs have emerged as an important molecules in the complex networks of gene regulation [3]. These naturally occurring small non coding RNA molecules that regulate the expression of protein coding genes at post-transcriptional level have been implicated in a variety of human disorders, such as infectious diseases, metabolic disease and malignancy [4]. Several hundred genes in our genome encode small functional RNA molecules collectively called miRNAs and are found in normal tissues, blood, and saliva.

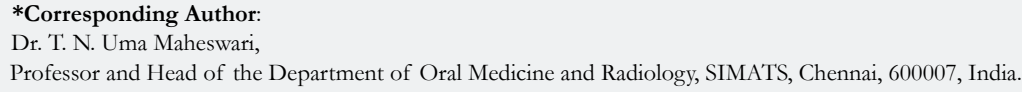

Citation: R. Amritha Sripoo, T. N. Uma Maheswari. Knowledge, Attitude And Perception On Genomic Markers In Oral Potentially Malignant Disorder Among Oral Medicine And Oral Pathology Specialists. Int J Dentistry Oral Sci. 2021;8(10):4842-4847. doi: http://dx.doi.org/10.19070/2377-8075-21000979

Copyright: T. N. Uma Maheswari ${ }^{\circ} 2021$. This is an open-access article distributed under the terms of the Creative Commons Attribution License, which permits unrestricted use, distribution and reproduction in any medium, provided the original author and source are credited. 
miRNA playsan important role in cellular growth, differentiation, apoptosis, and immune response [5]. During development of malignancy, some miRNAs are upregulatedand some are downregulated, so any change in the expression of miRNAs can cause tumor suppression. Apart from functioning as tumor suppressors, miRNAs can also promote tumor development (oncogenes) depending on the tumor types and their specific target protein $[5,6]$. The main role of microRNA in the human body is gene regulation. Given the low survival rate of multiple cancer types, credible biomarkers for cancer prognosis are urgently required [7].

In recent years, cancer has become the primary cause of mortality in most countries and regions, and the incidence of human malignancies has increased substantially [8]. Oral cancer is a preventable disease as it is mostly preceded by a group of precursor lesions called oral potentially malignant disorders (OPMDs) [9, 10]. Majority (up to $62 \%$ ) develop from a visible or invisible OPMD that is characterized by an increased risk for malignant transformation (MT) to OSCC [11]. According to the WHO, India is the second largest consumer and third largest producer of tobacco, increasing the incidence of OPMD in India which demands the researchers to involve in studies to find diagnostic biomarkers in detecting early malignant changes in OPMD.

Scully et al. proved clinical and histopathological assessment of OPMD is not sufficient to predict malignant transformation, hence, assessing the miRNA in these lesions would be helpful $(9,12)$. Cervigne et al. stated tissue expression of miRNA 21, miRNA $181 \mathrm{~b}$, and miRNA 345 is an early event in leukoplakia transforming into malignancy [9, 10, 12]. During development of malignancy, some miRNAs are upregulated and some are downregulated, so any change in the expression of miRNAs can cause tumor suppression or act as carcinogens [4]. Each miRNA can regulate expression of many target genes (multiple proteins) and expression of each target gene (specific protein) may also be regulated by multiple miRNAs [13]. miRNA was found to be deregulated in systemic diseases such as diabetes $[14,15]$ and hypertension [16] and was later found to be deregulated in ovarian [17], breast [18], colon [19], liver [19, 20], and pancreatic cancer tissues [21]. During the process of development of oral cancer, certain genes acquire roles in tumorigenesis while some are tumor suppressors. Increased levels of certain miRNAs cause progression of malignancy while some suppress malignancy. The upregulated salivary miRNA 184, and miRNA 21 and downregulated salivary miRNA 145 can be used as potential biomarkers to predict malig- nancy. Of these three, salivary miRNA 184 had the highest sensitivity and specificity [3]. The aim of this survey was to assess the knowledge, attitude and perception of genomic biomarkers among Oral medicine and Oral pathology specialists.

\section{Materials And Method}

This is a cross-sectional study based on self-reported questionnaires. An online questionnaire was developed by using google forms, with a consent form appended to it. Link of the questionnaire was shared through emails, whatsapp and other social media. Participants with access to the internet could participate in the study. Participants able to understand english language, who are pursuing or completed their master degree in Oral Medicine and Oral pathology and willing to give informed consent were included. Ethical approval for conducting the survey was obtained from the Saveetha Dental College and Hospitals. All the data were collected and compiled by the author. Simple random sampling was used to select the participants for the study. This provides equal odds for every member of the population to be chosen as a participant in the study. The primary items were reviewed by peers who provided feedback and suggested necessary changes in order to establish both face and content validity of the survey questionnaire.

Online self-reported questionnaires developed, which contained 12 multiple choice questions. They were asked to fill in the questionnaire individually. Responses were. Excel spreadsheets were used for data collection and manipulation. Age, gender, profession, knowledge, attitude and perception were assessed. Output was collected as nominal values, so percentage was calculated and the results were tabulated. Results were represented by pie charts and bar graphs

Data were analyzed using IBM SPSS software version 22. Data were described using frequencies and percentages. Chi-square was used to analyze differences between categorical variables. A pvalue of less than 0.05 was considered statistically significant.

\section{Results}

Study was conducted among 35 Oral Medicine specialists and Oral pathology specialists, out of which 12 were males (34\%) and $23(66 \%)$ were female (Table 1$)$ and 17 were Oral medicine specialist $(49 \%)$ and 18 were oral pathology specialist (51\%)(Table 2)

Table 1. Gender distribution.

\begin{tabular}{|c|c|c|}
\hline GENDER & FREQUENCY & PERCENTAGE \\
\hline MALES & 12 & $34 \%$ \\
\hline FEMALES & 23 & $66 \%$ \\
\hline
\end{tabular}

Table 1 showing frequency and percentage distribution of gender.

Table 2. Specialists.

\begin{tabular}{|c|c|c|}
\hline SPECIALISTS & FREQUENCY & PERCENTAGE \\
\hline ORAL MEDICINE \& RADIOLOGY & 17 & $49 \%$ \\
\hline ORAL PATHOLOGY & 18 & $51 \%$ \\
\hline
\end{tabular}

Table 2 showing frequency and percentage distribution of specialist 
46\% were first year MDS students, 29\% were second year MDS students and $25 \%$ were third year MDS students, given in Graph 1. $25 \%$ were readers and $75 \%$ were professors, given in Graph 2 . Among the oral medicine and oral pathology specialists, $17 \%$ had less than 5 years of practice, $42 \%$ had $5-10$ years of practice, $25 \%$ had 10-15 years of practice and 17\% had more than 15 years of clinical practice; given in Graph 3.

Graph 4 shows participants attitude on biomarker chosen for their research in OPMD.30 people $(86 \%)$ chose genomics for their research, $4(11.4 \%)$ on proteomics and $1(2.9 \%)$ on metabolomics.

Graph 5 shows genomic biomarker chosen for their research in OPMD. 27 people $(77.1 \%)$ has selected microRNA for their research, 6(17.1\%) selected Mt.DNA, 1(2.9\%) selected DNA and $1(2.9 \%)$ selected RNA.

Graph 6 shows how genomic biomarker selection was done for genomic biomarker research in OPMD. 13 people (37.1\%) selected based on performing a Systematic review, 7(20\%) selected based on Guide's previous research opinion and 15(42.9\%) selected based on previous literature research.

Graph 7 shows Samples used for genomic biomarker research in OPMD. 16 people (45.7\%) used Saliva, 14(40\%) used tissue and $5(14.3 \%)$ used Serum.

Graph 8 shows easy and convenient one for genomic biomarker research in OPMD. 22 people $(62.9 \%)$ suggested Saliva ,9(25.7\%) suggested tissue and 4(11.4\%) suggested Serum.
Graph 9 shows samples with better patient compliance for genomic biomarker research in OPMD. 22 people $(62.9 \%)$ suggested Saliva, $9(25.7 \%)$ suggested serum and 4(11.4\%) suggested tissue.

Graph 10 shows the participants knowledge on the most sensitive biomarker. 15 people (42.9\%) suggested miRNA 21, 9(25.7\%) suggested miRNA 184 and 11(31.4\%) suggested miRNA 31.

Graph 11 shows the participants perception on difficulties faced during genomic biomarker research in OPMD. 6 people $(17.1 \%)$ suggested Sample collection, 14(40\%) suggested Storage and Transport and 15(42.9\%) suggested processing of Sample.

Graph 12 shows statistical analysis involved in genomic biomarker research in OPMD. 6 (45.7\%) Sensitivity/Specificity, 11(31.4\%) AUC Curve and $8(22.9 \%)$ Cut off Value.

Graph 12 shows sample processing involved in genomic biomarker research in OPMD. 18(51.4\%) done under the guidance, $11(31.4 \%)$ observed alone and 6(17.1\%) performed.

Graph 14 shows sample size involved in genomic biomarker research in OPMD. 18(51.4\%) included a sample size of $>25$, $16(45.7 \%)$ included a sample size of $50-100$ and $1(2.9 \%)$ included a sample size of $<100$.

$42.9 \%$ revealed microRNA 21 as the most sensitive microRNA in OPMD(Graph 15), 49.2\% revealed easy and convenient method for Genomic biomarker research in OPMD as Saliva (Graph 16) and $43 \%$ revealed the major difficulty faced during Genomic biomarker research in OPMD is Processing of Samples (Graph 17).

\section{Graph 1. Year of study of PG students.}

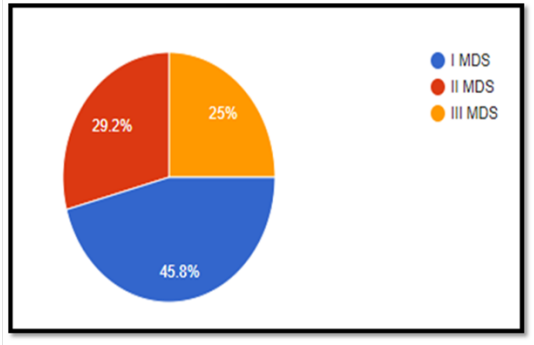

Graph 3. Years of practice of Practitioners.

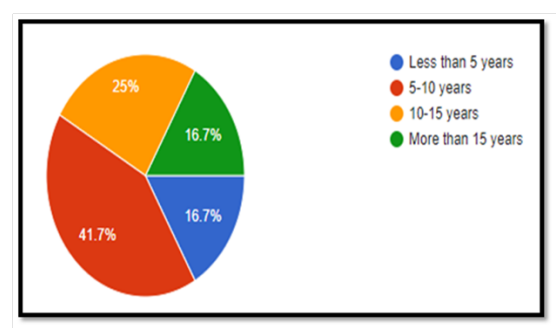

Graph 5. Genomic Biomarker for research in OPMD.

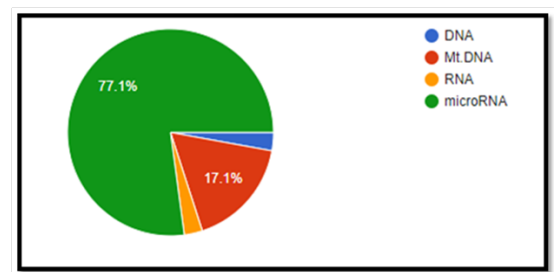

Graph 2. Designation of faculty member.

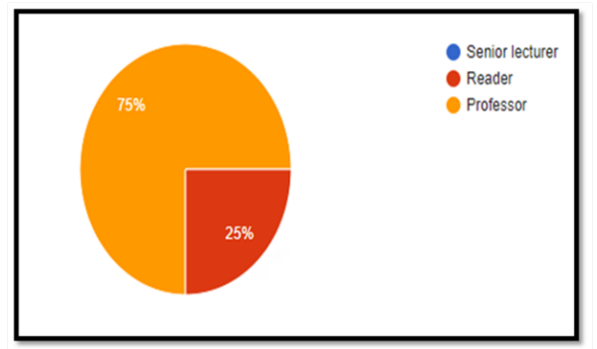

Graph 4. Biomarker for research in OPMD.

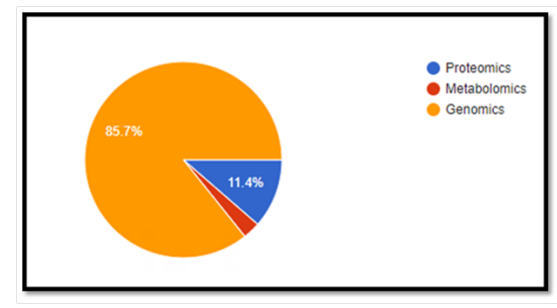

Graph 6. Genomic biomarker selection.

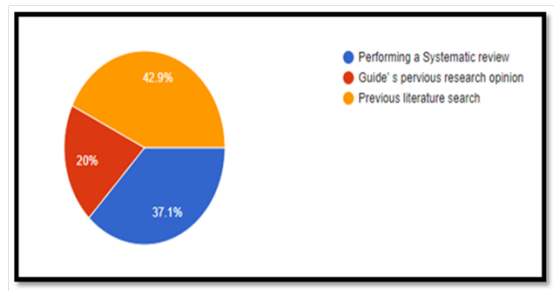


Graph 7. Sample used for research on Genomic markers in OPMD.

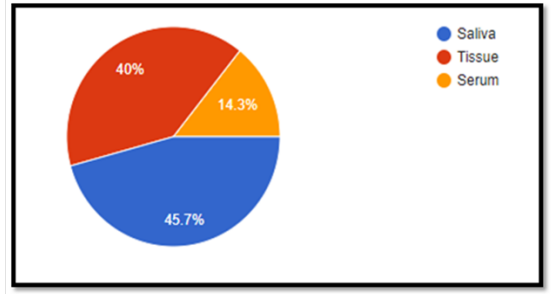

Graph 9. Better patient compliance.

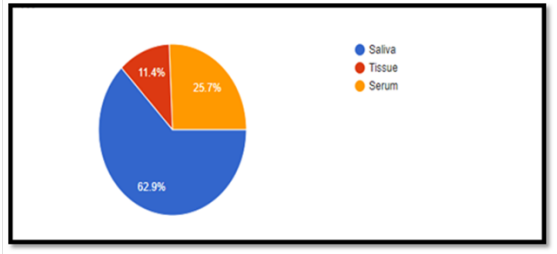

Graph 11. Difficulties faced during research.

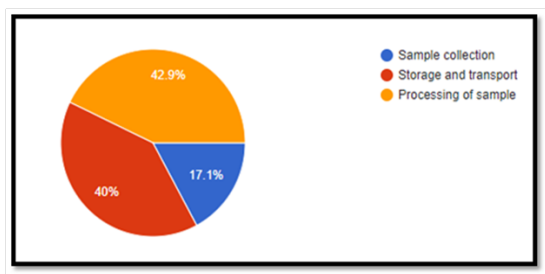

Graph 13. Sample processing.

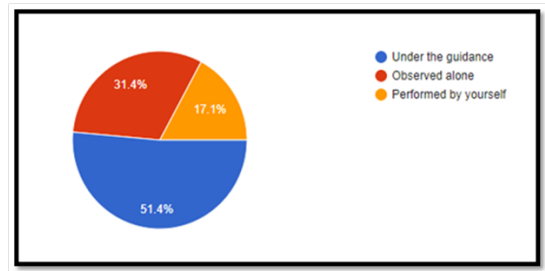

Graph 8. easy and convenient for research.

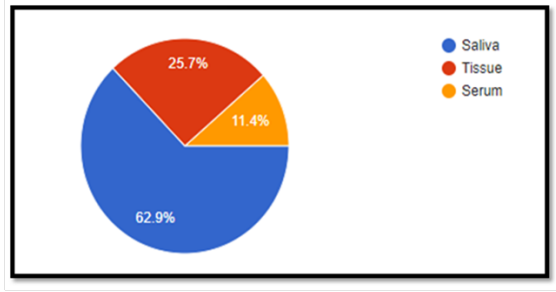

Graph 10. Most sensitive microRNA in OPMD.

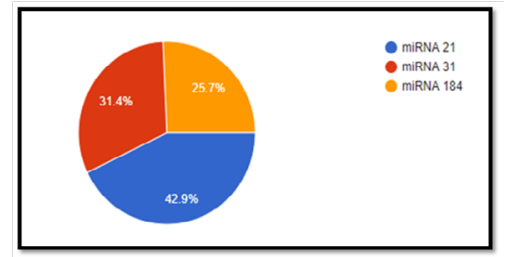

Graph 12. Statistical analysis was involved.

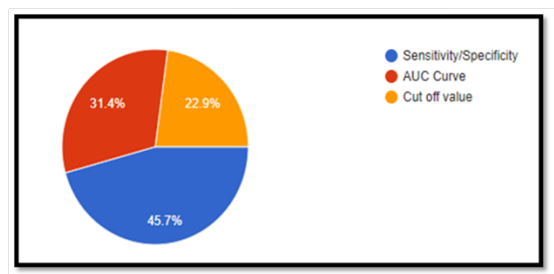

Graph 14. Sample size involved.

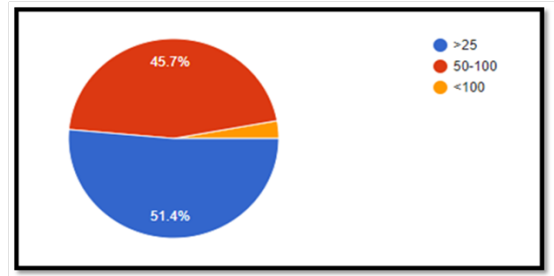

Graph 15. Represents Oral medicine and Oral Pathologists Knowledge towards the most sensitive microRNA in OPMD.

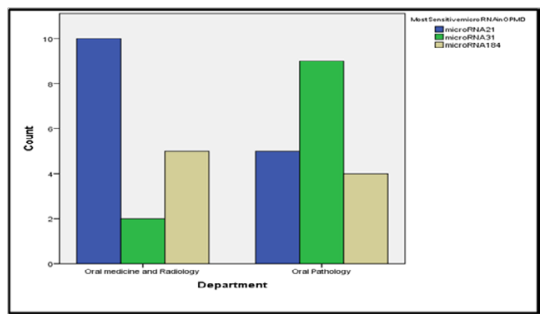

Graph 16. Represents Oral medicine and Oral Pathologists perception towards the difficulties faced during Genomic biomarker research in OPMD.

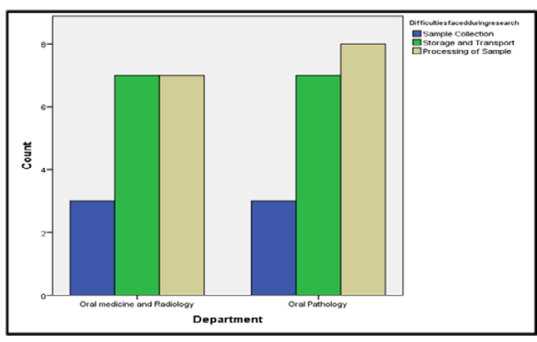

\section{Discussion}

This study was conducted among 35 Oral Medicine and Oral Pathology specialists who has done genomic biomarker research, out of which 12 were males $(34 \%)$ and $23(66 \%)$ were female and 17 were Oral medicine specialist $(49 \%)$ and 18 were oral pathology specialist (51\%). The results of our study showed that most of the participants had sufficient knowledge about genomic biomarkers and difficulties faced during genomic biomarker research in OPMD. Emerging evidence demonstrates an important role of miRNAs in regulating diverse cellular processes including 
Graph 17. Represents Oral medicine and Oral Pathologists Perception towards easy and Convenient method for Genomic biomarker in OPMD.

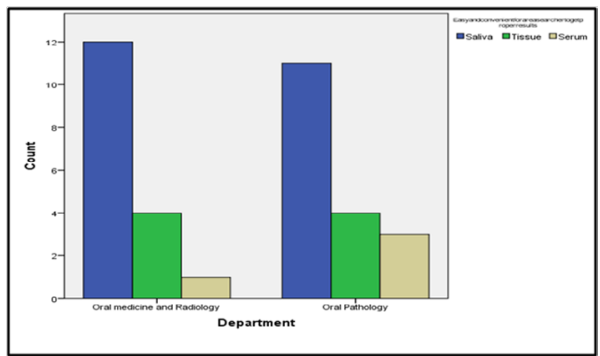

differentiation, proliferation and apoptosis [22]. Literature search revealed no other studies were conducted on knowledge, awareness and perception of Genomic biomarkers in OPMD. From the survey results only small sample size is involved in most studies. The contribution of miRNAs in various types of cancers differs. Therefore, it is essential to have a larger sample size to be able to decide between the healthy or diseased status. Saliva contains many enzymatic enzymes and hence the pathway identification is difficult whereas tissue is site specific and gives more accuracy when compared to saliva samples even though Saliva is easy and convenient and is patient compliance when compared to tissue. The results of Zahran et al. study revealed upregulatedmiRNA 184 with an area under the curve (AUC) of 0.86 and miRNA 21 with an AUC of 0.73 and downregulated miRNA 145 with an AUC of 0.68 , which proved that these miRNAs are significant in detecting early malignancy in OPMD and should be further analyzed in various populations [3]. There is an impervious need for faster ways to detect different pathologies, seeing that many types of cancer are discovered in late stage. miRNAs as biomarkers can fulfill this, thus being an impressive research field(13). The most important evaluation criteria for circulating miRNAs as diagnostic and prognostic biomarkers are high sensitivity and specificity, to avoid false positive or negative diagnosis. An appropriate biomarker for a specific cancer type should be both significantly differentially expressed and in correlation with the outcome of patients. Further, the identification of miRNAs and their target genes provides a novel insight into understanding the mechanisms of the tumor formation and progression, and promising therapy for different cancers (6). Majority of the participants believed that genomic biomarkers can serve as a diagnostic and prognostic tool in OPMD. Most of them had a positive attitude that it can be translated into routine clinical practice. Early detection of disease plays a crucial role for treatment planning and prognosis. The most common difficult faced by the researchers was processing of the samples. Since it was an online study, not many people from older age groups participated in the survey and the study was limited to the participants with access to the internet, who had smartphones and e-mail IDs. And the survey was in english so people who understand english could only participate. This survey provides an insight for future researches to fill up the existing gaps.

\section{Conclusion}

Oral medicine and Oral pathology specialists have adequate awareness and knowledge aboutgenomic biomarkers in Oral Potentially malignant disorders, but certain knowledge has to be brushed up among them. Majority of participants showed a positive attitude towards its use as a diagnostic \& prognostic tool. Fur- thermore, they need to be trained on these grounds to help them treat their patients in the best possible way. As current techniques evolve, we anticipate that miRNAs will become a routine approach in the development of personalized patient profiles, therefore allowing targeted therapeutic interventions.

\section{References}

[1]. Institute NC, National Cancer Institute. Genomic Biomarker [Internet]. Definitions. 2020.

[2]. Perkel JM. Genomic Biomarker Discovery: Bringing the Genome to Life [Internet]. Science. 2008.

[3]. Maheswari TNU, Venugopal A, Sureshbabu NM, Ramani P. Salivary micro RNA as a potential biomarker in oral potentially malignant disorders: A systematic review. CiJi Yi XueZaZhi. 2018 Apr-Jun;30(2):55-60. PubmedPMID: 29875583.

[4]. Wu W. MicroRNA and Cancer: Methods and Protocols. Humana Press; 2017. $222 \mathrm{p}$.

[5]. Iorio MV, Croce CM. microRNA involvement in human cancer. Carcinogenesis. 2012 Jun 1;33(6):1126-33.

[6]. El-Sakka H, Kujan O, Farah CS. Assessing miRNAs profile expression as a risk stratification biomarker in oral potentially malignant disorders: A systematic review. Oral Oncol. 2018 Feb;77:57-82. PubmedPMID: 29362128.

[7]. Zhang J, Xu A, Miao C, Yang J, Gu M, Song N. Prognostic value of Lin28A and Lin28B in various human malignancies: a systematic review and metaanalysis. Cancer Cell Int. 2019 Apr 2;19:79. PubmedPMID: 30976203.

[8]. Bray F, Ren JS, Masuyer E, Ferlay J. Global estimates of cancer prevalence for 27 sites in the adult population in 2008. Int J Cancer. $2013 \mathrm{Mar}$ 1;132(5):1133-45. PubmedPMID: 22752881

[9]. Sankaranarayanan R, Ramadas K, Amarasinghe H, Subramanian S, Johnson N. Oral Cancer: Prevention, Early Detection, and Treatment. In: Gelband H, Jha P, Sankaranarayanan R, Horton S, editors. Cancer: Disease Control Priorities, Third Edition (Volume 3). Washington (DC): The International Bank for Reconstruction and Development / The World Bank; 2015 Nov 1. Chapter 5. PubmedPMID: 26913350.

[10]. Cervigne NK, Reis PP, Machado J, Sadikovic B, Bradley G, Galloni NN, et al. Identification of a microRNA signature associated with progression of leukoplakia to oral carcinoma. Hum Mol Genet. 2009 Dec 15;18(24):481829. PubmedPMID: 19776030.

[11]. Elimairi I, Sami A, Yousef B. Oral Cancer and Potentially Malignant Disorders [Internet]. Histopathology - An Update. 2018.

[12]. Scully C. Challenges in predicting which oral mucosal potentially malignant disease will progress to neoplasia. Oral Dis. 2014 Jan;20(1):1-5. PubmedPMID: 24320967.

[13]. Chattopadhyay E, Singh R, Ray A, Roy R, De Sarkar N, Paul RR, et al. Expression deregulation of mir31 and CXCL12 in two types of oral precancers and cancer: importance in progression of precancer and cancer. Sci Rep. 2016 Sep 6;6:32735. PubmedPMID: 27597234.

[14]. McClelland AD, Kantharidis P. MicroRNAs as Biomarkers of Diabetic Nephropathy [Internet]. Biomarkers in Kidney Disease. 2015. p. 1-29.

[15]. Chung AC. microRNAs in Diabetic Kidney Disease. Adv Exp Med Biol. 2015;888:253-69. PubmedPMID: 26663187.

[16]. Marques FZ, Charchar FJ. microRNAs in Essential Hypertension and Blood Pressure Regulation. Adv Exp Med Biol. 2015;888:215-35. PubmedPMID: 26663185.

[17]. Orio MV, Visone R, Di Leva G, Donati V, Petrocca F, Casalini P, et al. MicroRNA signatures in human ovarian cancer. Cancer Res. 2007 Sep 15;67(18):8699-707. PubmedPMID: 17875710.

[18]. Yen MC, Shih YC, Hsu YL, Lin ES, Lin YS, Tsai EM, et al. Isolinderalactone enhances the inhibition of SOCS3 on STAT3 activity by decreasing miR- 
30c in breast cancer. Oncol Rep. 2016 Mar;35(3):1356-64. PubmedPMID: 26707189.

[19]. Mullany LE, Wolff RK, Herrick JS, Buas MF, Slattery ML. SNP Regulation of microRNA Expression and Subsequent Colon Cancer Risk. PLoS One. 2015 Dec 2;10(12):e0143894. PubmedPMID: 26630397.

[20]. Perilli L, Pizzini S, Bisognin A, Mandruzzato S, Biasiolo M, Facciolli A, et al. Human miRNome profiling in colorectal cancer and liver metastasis de- velopment. Genom Data. 2014 Jun 27;2:184-8. PubmedPMID: 26484092.

[21]. Lau C, Kim Y, Chia D, Spielmann N, Eibl G, Elashoff D, et al. Role of pancreatic cancer-derived exosomes in salivary biomarker development. J Biol Chem. 2013 Sep 13;288(37):26888-97. PubmedPMID: 23880764.

[22]. Croce CM, Calin GA. miRNAs, cancer, and stem cell division. Cell. 2005 Jul 15;122(1):6-7. PubmedPMID: 16009126. 